\title{
Energy dependence of quantum dot formation by ion sputtering
}

\author{
S. Facsko and H. Kurz \\ Institute of Semiconductor Electronics, Rheinisch-Westfälische Technische Hochschule Aachen, Sommerfeldstrasse 24, \\ 52056 Aachen, Germany \\ T. Dekorsy \\ Institut für Ionenstrahlphysik und Materialforschung, Forschungszentrum Rossendorf, Postfach 510119, 01314 Dresden, Germany
}

\begin{abstract}
Ordered quantum dot patterns are generated on GaSb and InSb surfaces due to a surface instability induced by $\mathrm{Ar}^{+}$-ion sputtering at normal incidence. The characteristic length of the generated patterns scales with the square root of the ion energy over the energy range of 75-1800 eV. This energy dependence is compared to the solutions of the isotropic Kuramoto-Sivashinsky equation and allows the determination of the lateral width of the energy distribution deposited by the incident ions in the very-low-energy range. We show that the observed energy dependence is in agreement with the linear continuum theory under the assumption that the dominant smoothing process is due to effective ion-induced diffusion without mass transport on the surface.
\end{abstract}

The controlled fabrication of semiconductor nanostructures remains a key requirement for the development of future optoelectronic and electronic devices. Although electron-beam lithography enables the fabrication of structures with nanometer dimensions, the low throughput of serial lithographic methods represents a severe bottleneck. Therefore alternative methods based on self-organization to form large arrays of well-defined quantum dots (QD's) have attracted extensive attention in the past. Up to now most of the work was based on the spontaneous growth of selforganized QD's on semiconductor surfaces in the StranskiKrastanov (SK) growth mode. ${ }^{1}$ Very recently, low-energy ion sputtering at normal incidence has been demonstrated to be an attractive alternative to SK growth, opening a promising route for the parallel fabrication of uniform semiconductor QD's ordered in a hexagonal array. ${ }^{2}$ This self-organized formation of periodic arrays of QD's during ion sputtering is based on a surface instability, i.e., the interplay between surface roughening induced by sputtering and smoothing processes on the surface. The basic mechanism has been related to physical principles underlying the formation of periodic ripple structures during ion sputtering of surfaces at oblique angles of incidence. ${ }^{3}$ These ripple patterns have been observed on different surfaces of semiconductors, ${ }^{4-8}$ metals,,${ }^{9,10}$ and glass. ${ }^{11}$ The formation of ripple patterns has been investigated experimentally and theoretically in great detail ${ }^{12}$ while the formation of regular dot arrays is not yet fully reconnoitered.

In order to control the formation process for the QD's induced by ion sputtering a quantitative understanding of the formation mechanism is required. For the description of the dot formation mechanism a continuum model has been developed, which is related microscopically to the distribution function of the energy deposited by the incident ions, ${ }^{3}$ and allows the derivation of the wavelength of the pattern. Other microscopic models relate the formation of nanostructures to the formation of surface defects and adatoms and their diffusion on the surface. ${ }^{10,13}$ However, quantitative predictions are difficult to extract from these models. Here we report on the investigation of the energy dependence of the dot evolution during low-energy ion sputtering. We observe a squareroot dependence of the characteristic length of the generated dot patterns over a large energy range. This energy dependence confirms the predictions of the continuum model for dot patterns under isotropic sputtering conditions and allows us to draw conclusions on the type of smoothing process involved. We find that the scaling with energy of the characteristic length is directly related to the scaling of the lateral width of the energy deposited by the incident ions, thus allowing the experimental verification of this parameter in the low-energy regime.

The evolution of the surface during the sputter process is described by a partial differential equation, ${ }^{3}$ which incorporates the characteristic dependence of the erosion rate on the local surface curvature. ${ }^{14}$ Under certain conditions a surface instability is induced with a roughening rate proportional to the second derivative of the surface contour. ${ }^{3,12,15,16}$ The resulting equation describing the dynamics of the surface morphology was originally considered for the description of chemical waves and flame fronts and is known as the Kuramoto-Sivashinsky (KS) equation. ${ }^{17,18}$ In the special case of isotropic ion sputtering, i.e., at normal incidence or nonnormal incidence in conjunction with a rotating surface, the isotropic KS equation describes the temporal evolution of the surface height function $h(x, y)$ as ${ }^{15,19}$

$$
\frac{\partial h(x, y)}{\partial t}=v_{0}+\nu \nabla^{2} h-D \nabla^{2}\left(\nabla^{2} h\right)+\frac{\lambda}{2}(\nabla h)^{2}+\eta(x, y, t),
$$

with

$$
\begin{gathered}
\nu=-\frac{a \mu^{2}}{2 \sigma^{2}}\left[\frac{J \epsilon p}{\sqrt{2 \pi} \sigma} \exp \left(-\frac{a^{2}}{2 \sigma^{2}}\right)\right], \\
\lambda=\frac{\mu^{2}}{2}\left(\frac{1}{\sigma^{2}}-\frac{a^{2}}{\sigma^{4}}-\frac{1}{\mu^{2}}\right)\left[\frac{J \epsilon p}{\sqrt{2 \pi} \sigma} \exp \left(-\frac{a^{2}}{2 \sigma^{2}}\right)\right] .
\end{gathered}
$$


Here $v_{0}$ is the average erosion velocity of the surface during sputtering, $\nu$ is the effective surface tension induced by ion sputtering, $D$ is the coefficient related to relaxation by surface diffusion which will be explained in detail below, $\lambda$ is the tilt-dependent erosion rate, and $\eta(x, y, t)$ is white noise incorporating the stochastic nature of the ion-sputtering process. The coefficients $\nu$ and $\lambda$ are calculated from the distribution of the energy deposited by the incident ions, which is assumed to be Gaussian, as given in Eqs. (2) and (3) (for details see Refs. 3, 12, and 20). They are functions of ion current density $J$, ion energy $\epsilon$, and of the parameters describing the Gaussian energy distribution, i.e., ion range $a$, longitudinal width $\sigma$, and lateral width $\mu . p$ is the proportionality factor coupling the energy deposited with the erosion rate.

The surface relaxation due to diffusion is described by the term $D \nabla^{2}\left(\nabla^{2} h\right)$ in Eq. (1). This term may be governed by different physical processes that may or may not involve mass transport on the surface. Originally, the surface diffusion was introduced as the thermally activated surface diffusion $D_{t}=D_{0} \exp \left(-E a / k_{B} T\right)$, with $E_{a}$ the activation energy, $k_{B}$ Boltzmann's constant, and $T$ the temperature. ${ }^{3}$ This smoothing rate is based on mass transport on the surface. Going beyond the second-order term in the series expansion of the erosion velocity an additional term must be taken into account, with the same mathematical form $D_{\text {eff }} \nabla^{2}\left(\nabla^{2} h\right)$ as the thermal diffusion but of different origin. $D_{\text {eff }}$ is called effective ion-induced surface diffusion and must be regarded as a smoothing contribution in the morphology evolution without mass transport. ${ }^{20}$ The coefficient $D_{\text {eff }}$ is directly related to the parameters of the distribution of the energy deposited,

$$
D_{\text {eff }}=\frac{a \mu^{4}}{8 \sigma^{2}}\left[\frac{J \epsilon p}{\sqrt{2 \pi} \sigma} \exp \left(-\frac{a^{2}}{2 \sigma^{2}}\right)\right] .
$$

The total smoothing rate is the sum of the two diffusion terms $D_{t}$ and $D_{\text {eff }}$. At low temperature and high ion energy the effective ion-induced surface diffusion should clearly dominate over thermal diffusion.

Periodic surface structures are already predicted by the linear form of Eq. (1), i.e., $\lambda=0$. A negative effective surface tension $(\nu<0)$ introduces a surface instability that tends to increase the surface roughness but which is balanced by surface smoothing via diffusion. ${ }^{3}$ A Fourier analysis of the solutions of the linear equation yields periodic structures with a characteristic wavelength $l_{c}=2 \pi \sqrt{2 D /|\nu|}$. Amplitudes with such a periodicity grow exponentially with time.

The nonlinear term $\lambda / 2(\nabla h)^{2}$ in Eq. (1) becomes relevant for later times at higher slopes of the height function $h(x, y)$. It has been demonstrated recently by numerical analysis of the KS equation that the nonlinear term leads to a saturation of the amplitude of the periodic structures. ${ }^{21}$ In addition, this term governs the formation of uniform and long-range hexagonal ordering. This self-ordering appears in the early stage of the nonlinear regime with the same characteristic length $l_{c}$ as given by the solution of the linear equation. Without this nonlinear term solutions with fourfold symmetric dot pat- terns are expected only. For longer times the surface contour evolves towards unordered patterns and enters the regime of kinetic roughening. ${ }^{21}$

The main part of this paper is devoted to investigating the role of ion-induced diffusion for the formation of QD patterns, as manifested in the energy dependence of the pattern period. In the case of ion-induced diffusion the characteristic wavelength $l_{c}$ obtained from Eqs. (2) and (4) is

$$
l_{c}=\sqrt{2} \pi \mu .
$$

From sputter theory it is known that the lateral width $\mu$ of the energy deposited varies with

$$
\mu \sim \epsilon^{2 m},
$$

with $m$ ranging from $m=1$ at high energies down to $m \approx 0$ at very low energies. ${ }^{22,23}$ The parameter $m$ originates from the interatomic potential $V(R)=R^{-1 / m}$ determining the stopping cross section. At very high ion energies Rutherford scattering with $m=1$ is assumed. At low energies the screening of the Coulomb interaction becomes essential decreasing $m$ to $\sim 1 / 2$ over several $\mathrm{keV}$ range and to $\sim 1 / 3$ in the lower $\mathrm{keV}$ range. ${ }^{22}$ In the low-energy range considered in our experiments the characteristic length should therefore increase with ion energy according to the power law in Eq. (6) with 0 $\leqslant m \leqslant 1 / 2$. Furthermore, $l_{c}$ should be independent of ion current density and sample temperature in the case of ioninduced diffusion. In distinct contrast to this ion-induced diffusion processes, thermally activated diffusion would lead to $\epsilon^{-m}$ dependence of the characteristic wavelength $l_{c}$. In addition, $l_{c}$ would depend on temperature and on ion current density. ${ }^{21}$

Here, the period of the QD patterns that are produced on (100) surfaces of GaSb and InSb wafers by low-energy $\mathrm{Ar}^{+}$ sputtering under normal incidence is carefully determined as a function of ion energy. The $\mathrm{Ar}^{+}$ions are generated in an electron cyclotron resonance (ECR) plasma and extracted by an accelerator voltage from the plasma to the sample surface. The $\mathrm{Ar}^{+}$ions bombard the surface at normal incidence with an ion current density of $2.5 \times 10^{15} \mathrm{~cm}^{-2} \mathrm{~s}^{-1}$. The average erosion velocity of the GaSb surface ranges from 0.2 to $2.6 \mathrm{~nm} \mathrm{~s}^{-1}$ for ion energies of 50 to $1800 \mathrm{eV}$, respectively. The sample temperature is kept constant during the ion sputtering at two distinct values of $-60^{\circ}$ and $+60^{\circ} \mathrm{C}$ by cooling the sample holder with water or liquid nitrogen. The surface morphology induced by the ion bombardment is analyzed $e x$ situ by scanning electron microscopy (SEM) and atomic force microscopy (AFM). The characteristic length of the periodicity and the QD size and density are extracted from the SEM images. In order to calculate the coefficients $\nu, D_{\text {eff }}$, and $\lambda$ independently, the parameters of the distribution of the energy deposited, which is assumed Gaussian, are calculated by a Monte Carlo simulation of the ionbombardment process in a binary collision approximation by the TRIM program. ${ }^{24}$

The time evolution of the QD's can be summarized as follows: ${ }^{2}$ after a short sputtering time of approximately $40 \mathrm{~s}$ small dots can already be identified with a characteristic length that depends on ion energy. During the subsequent 
sputtering the characteristic length decreases slightly, by approximately $20 \%$, whereas the dot diameter increases until the dots touch each other. In this stage the dots have a hexagonal closed-packed arrangement on the surface and undergo no further changes in the morphology during subsequent sputtering. The steady-state morphology observed in our experiments supports the experimentally observed saturation of the amplitude of ripple patterns. ${ }^{25}$ Besides the previously mentioned nonlinear term in the KS equation, different mechanisms have been proposed for the amplitude saturation. ${ }^{26,25}$

The characteristic wavelength $l_{c}$ was determined for ion current densities in the range of $1 \times 10^{15}-4$ $\times 10^{15} \mathrm{~cm}^{-2} \mathrm{~s}^{-1}$ and sample temperatures of $-60^{\circ} \mathrm{C}$ and $+60^{\circ} \mathrm{C}$ at an ion energy of $500 \mathrm{eV}$. The wavelength shows no dependence on ion current density and sample temperature. This observation is a clear hint that the relevant smoothing process is nonthermal up to $+60^{\circ} \mathrm{C}$ sample temperature at the ion energies used in the experiments. It supports the assumption that the effective ion-induced diffusion dominates over thermal diffusion in this temperature range in the dot formation process on $\mathrm{GaSb}^{21}$

Figure 1 shows QD patterns on GaSb surfaces after ion sputtering at energies of 100,450 , and $1500 \mathrm{eV}$, respectively. The ion dose was calculated from the sputtering yield to give a constant depth of the sputtered area of approximately 500 $\mathrm{nm}$. The regular long-range hexagonal ordering is present in all QD patterns. The insets show the corresponding distributions of the nearest-neighbor distance. At lower energies the dots have a circular and uniform shape and are ordered in an array with a narrow distribution of the nearest-neighbor distance [Figs. 1(a) and 1(b)]. At higher energies the shape of the dots becomes asymmetric with a larger spread in the distribution of their distance. This stage may indicate that the process enters the kinetic roughening regime for longer sputtering [Fig. 1(c)].

Figure 2 shows a double logarithmic plot of the characteristic wavelength $l_{c}$ for GaSb and InSb QD patterns versus the ion energy $\epsilon$. The data for the characteristic length for $\mathrm{GaSb}$ patterns shows two regimes in the measured energy range of 40 to $1800 \mathrm{eV}$. From $1800 \mathrm{eV}$ down to $75 \mathrm{eV}$ a power-law dependence of $l_{c}$ on $\epsilon$ with an exponent of 0.5 \pm 0.02 is determined. This result is in excellent agreement with the predictions for the characteristic length with effective ion-induced diffusion as the dominant smoothing process. Thus, the lateral width of the distribution of the deposited energy $\mu$ increases according to Eq. (6) with $m=0.25$. Below $75 \mathrm{eV}$ the measured value for $l_{c}$ rises again. For the three data points an exponent of -1.2 is determined. This rise may be either due to an increased influence of thermal diffusion challenging the effective ion-induced diffusion or due to the fact that at these low energies $m$ approaches zero, resulting in a constant width $\mu$ in Eq. (6).

The power-law dependence of $l_{c}$ on $\epsilon$ is also confirmed by the three data points obtained for InSb. At the same energy the characteristic length of the patterns generated on InSb surfaces are higher by a factor of 1.2 compared to $\mathrm{GaSb}$.
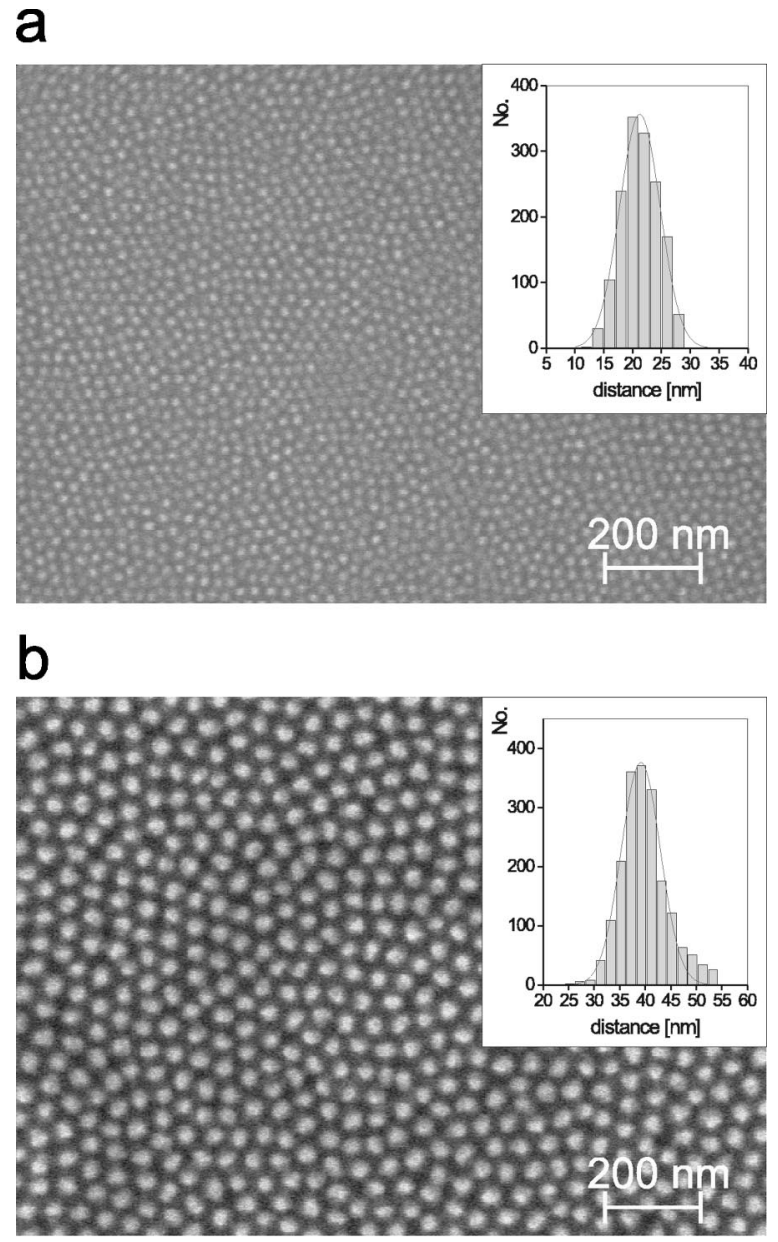

C

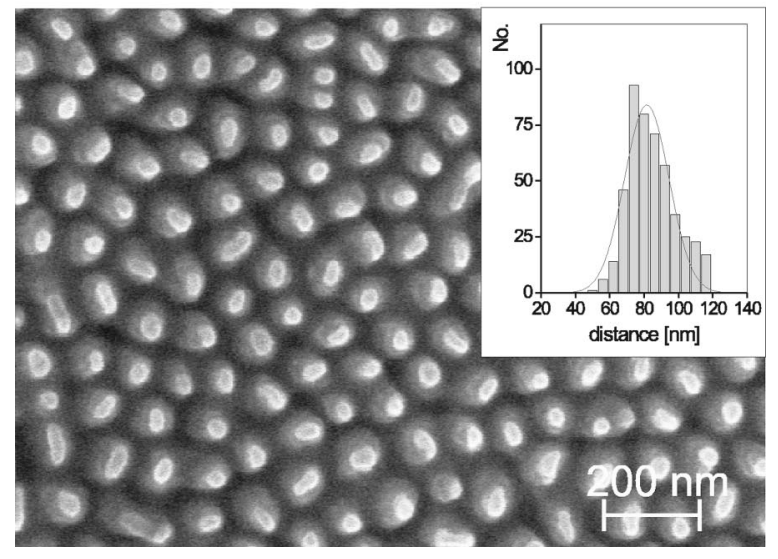

FIG. 1. Scanning electron micrographs of quantum dot patterns on $\mathrm{GaSb}$ surfaces induced by $\mathrm{Ar}^{+}$-ion sputtering with ion energies of (a) $100 \mathrm{eV}$, (b) $500 \mathrm{eV}$, and (c) $1500 \mathrm{eV}$. The dots show a hexagonal ordering with a characteristic wavelength that depends on ion energy. The insets show the corresponding distribution of the nearest-neighbor distance.

In Fig. 2 the calculated values for the characteristic wavelength $l_{c}=\sqrt{2} \pi \mu$ with $\mu$ simulated with TRIM are also shown. The values show the same power-law dependence between the characteristic length and $\epsilon$. There is a factor of 6 discrepancy between the calculated values and those ob- 


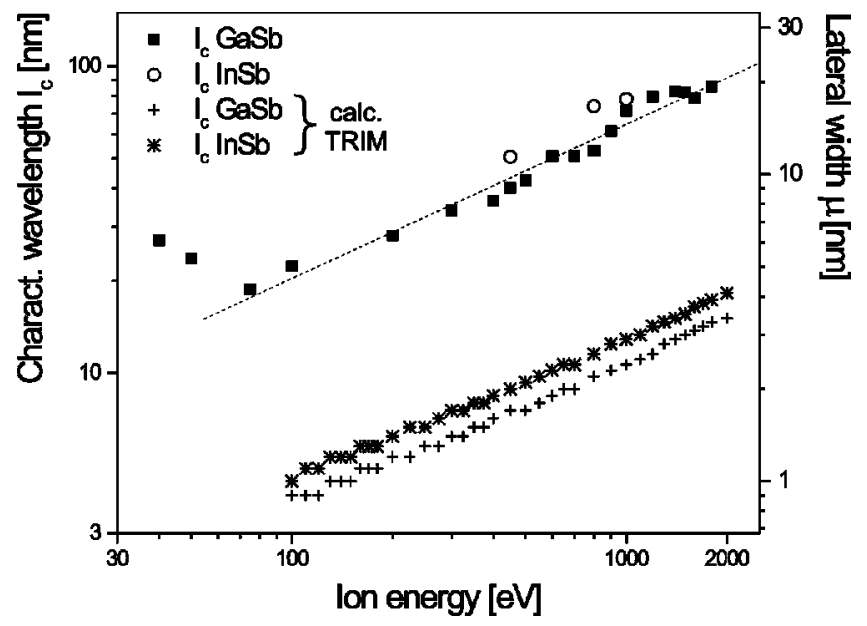

FIG. 2. Energy dependence of the characteristic wavelength $l_{c}$ of the QD patterns on $\mathrm{GaSb}(100)$ (squares) and $\mathrm{InSb}(100)$ (circles). The linear fit is shown as a dashed line for $\mathrm{GaSb}$ and gives an exponent of $0.50 \pm 0.02$ for the power-law dependence. The characteristic wavelength for $\mathrm{GaSb}(+)$ and $\operatorname{InSb}(\times)$ calculated with parameters simulated by TRIM are also shown.

tained experimentally. However, the change in characteristic length by a factor of 1.2 between $\mathrm{GaSb}$ and $\mathrm{InSb}$ in the experiments is well reproduced by the calculation giving a factor of 1.19. We attribute the factor of 6 discrepancy to an underestimate of the lateral width $\mu$ of the energy distribution by TRIM. TRIM is well known to have a high accuracy for high ion energies, ${ }^{24}$ but at low energies a higher error is expected due to many-body effects. Careful calculations of the energy distribution by molecular-dynamic simulations are necessary to clarify to what extent $\mu$ is underestimated by TRIM.

For oblique incidence ion sputtering the energy dependence of the characteristic wavelength has been investigated by several groups. ${ }^{6,27}$ Theoretically the characteristic wavelength is obtained from the more complicated anisotropic KS equation, which makes the results more sensitive to specific parameter differences than under isotropic sputtering conditions. e.g., Vajo et al. investigated the energy dependence of the characteristic wavelength for $\mathrm{Si}$ (100) surfaces in the energy range of 1.5 to $7 \mathrm{keV} .{ }^{27}$ They found that the ripple wavelength is independent of ion current density. Furthermore, a power-law dependence with a power of 0.8 on ion energy was determined, in agreement with the energy dependence for $\sigma$ and $\mu$, in the higher energy range. Furthermore, they found a ripple wavelength that scales as 40 times the ion range, which is also higher than the theoretical expected value.

In conclusion, we investigated the energy dependence of the characteristic wavelength of hexagonal dot arrays produced by low-energy ion sputtering under normal incidence. $\mathrm{On} \mathrm{GaSb}$ and InSb surfaces the characteristic length is proportional to the square root of the ion energy over a large range of energies. The energy dependence of the characteristic wavelength $l_{c}$ confirms predictions made by solutions of the linear continuum equation with an effective ion-induced diffusion as the dominant relaxation process for $\mathrm{Ar}^{+}$-ion energies of 75 to $1800 \mathrm{eV}$ and temperatures $\leqslant 60^{\circ} \mathrm{C}$. From the scaling of the characteristic length with ion energy the power-law dependence of the lateral width of the deposited energy with an exponent $2 m$ with $m=0.25$ is concluded.

The authors would like to thank C. Moormann and C. Zanke for SEM analysis, S. Hu and S. Hamidi for AFM measurements, M. Luysberg for TEM analysis, and to acknowledge valuable discussion with A.-L. Barabási and R. Cuerno. The authors thank T. Bobek for numerical analysis of the continuum equations and extensive discussions. T.D. acknowledges the financial support of North-Rhine Westphalia.
${ }^{1}$ V. A. Shchukin and D. Bimberg, Rev. Mod. Phys. 71, 1125 (1999).

${ }^{2}$ S. Facsko, T. Dekorsy, C. Koerdt, C. Trappe H. Kurz, A. Vogt, and H. L. Hartnagel, Science 285, 1551 (1999).

${ }^{3}$ R. M. Bradley and J. M. E. Harper, J. Vac. Sci. Technol. A 6, 2390 (1988).

${ }^{4}$ G. Carter, M. J. Nobes, H. Stoere, and I. V. Katardjiev, Surf. Interface Anal. 20, 90 (1993).

${ }^{5}$ E. Chason, T. M. Mayer, B. K. Kellerman, D. T. McIlroy, and A. J. Howard, Phys. Rev. Lett. 72, 3040 (1994).

${ }^{6}$ A. Karen, Y. Nakagawa, M. Okuno, F. Soeda, and A. Ishitani, Surf. Interface Anal. 23, 506 (1995).

${ }^{7}$ C. M. Demanent, J. B. Malherbe, N. G. van der Berg, and V. Sankar, Surf. Interface Anal. 23, 433 (1995).

${ }^{8}$ J. Erlebacher, M. J. Aziz, E. Chason, M. B. Sinclair, and J. A. Floro, Phys. Rev. Lett. 82, 2330 (1999).

${ }^{9}$ S. Rusponi, C. Boragno, and U. Valbusa, Phys. Rev. Lett. 78, 2795 (1997).

${ }^{10}$ S. Rusponi, G. Costantini, F. Bautier de Mongeot, C. Boragno, and U. Valbusa, Appl. Phys. Lett. 75, 3318 (1999).

${ }^{11}$ T. M. Mayer, E. Chason, and A. J. Howard, J. Appl. Phys. 76, 1633 (1994).

${ }^{12}$ M. Makeev, R. Cuerno, and A.-L. Barabási, cond-mat/0007354 (unpublished).

${ }^{13}$ T. Michely and C. Teichert, Phys. Rev. B 50, 11156 (1994).

${ }^{14}$ P. Sigmund, J. Mater. Sci. 8, 1545 (1973).

${ }^{15}$ R. Cuerno and A.-L. Barabási, Phys. Rev. Lett. 74, 4746 (1995).

${ }^{16}$ G. Carter, Phys. Rev. B 59, 1669 (1998).

${ }^{17}$ Y. Kuramoto and T. Suzuki, Prog. Theor. Phys. 55, 356 (1977).

${ }^{18}$ G. I. Sivashinsky, Acta Astron. 4, 1177 (1977).

${ }^{19}$ R. Cuerno, H. A. Makse, S. Tomassone, S.T. Harrington, and H. E. Stanley, Phys. Rev. Lett. 75, 4464 (1995).

${ }^{20}$ M. A. Makeev and A.-L. Barabási, Appl. Phys. Lett. 71, 2800 (1997).

${ }^{21}$ B. Kahng, H. Jeong, and A.-L. Barabási, Appl. Phys. Lett. 78, 805 (2001).

${ }^{22}$ P. Sigmund, Phys. Rev. 184, 383 (1969).

${ }^{23}$ P. Sigmund, in Sputtering by Particle Bombardment I, edited by 
R. Behrish, Topics in Applied Physics Vol. 47 (Springer-Verlag, Berlin, 1981), Chap. 1, pp. 8-71.

${ }^{24}$ J. F. Ziegler, J. P. Biersack, and U. Littmark, The Stopping and Range of Ions in Matter (Pergamon Press, New York, 1985).

${ }^{25}$ J. Erlebacher and M. J. Aziz, J. Vac. Sci. Technol. A 18, 115 (2000).
${ }^{26}$ M. V. Ramana Murty, T. Curcic, A. Judy, B. H. Cooper, A. R. Woll, J. D. Brock, S. Kycia, and R. L. Headrick, Phys. Rev. Lett. 80, 4713 (1998).

${ }^{27}$ J. J. Vajo, R. E. Doty, and E.-H. Cirlin, J. Vac. Sci. Technol. A 14, 2709 (1996). 